\title{
Localized Waves in Optical Systems with Periodic Dispersion and Nonlinearity Management
}

\author{
Brandon G. Bale, ${ }^{1}$ Sonia Boscolo, ${ }^{1}$ Osip Y. Schwartz, ${ }^{2}$ and Sergei K. Turitsyn ${ }^{1}$ \\ ${ }^{1}$ Photonics Research Group, School of Engineering and Applied Science, Aston University, Birmingham B4 7ET, UK \\ ${ }^{2}$ Weizmann Institute of Science, Rehovot, 76100, Israel \\ Correspondence should be addressed to Sonia Boscolo, s.a.boscolo@aston.ac.uk
}

Received 26 March 2009; Accepted 29 June 2009

Recommended by J. Kutz

We overview our recent developments in the theory of dispersion-managed (DM) solitons within the context of optical applications. First, we present a class of localized solutions with a period multiple to that of the standard DM soliton in the nonlinear Schrödinger equation with periodic variations of the dispersion. In the framework of a reduced ordinary differential equation-based model, we discuss the key features of these structures, such as a smaller energy compared to traditional DM solitons with the same temporal width. Next, we present new results on dissipative DM solitons, which occur in the context of mode-locked lasers. By means of numerical simulations and a reduced variational model of the complex Ginzburg-Landau equation, we analyze the influence of the different dissipative processes that take place in a laser.

Copyright ( 2009 Brandon G. Bale et al. This is an open access article distributed under the Creative Commons Attribution License, which permits unrestricted use, distribution, and reproduction in any medium, provided the original work is properly cited.

\section{Introduction}

Nonlinear distributed systems with periodic variations of one or several key parameters present a very important branch of nonlinear science with a range of practical applications in solid-state physics, optics, plasma physics, hydrodynamics, wave physics and other fields. The impacts of nonlinear effects on wave propagation or modifications of the nonlinear wave properties by a medium with periodically varying parameters are two important fundamental problems that have been actively studied in the past decades. It is well accepted nowadays that very often, nonlinear models governing rather different physical phenomena engaging nonlinearity and periodicity of a medium characteristics can be quite similar mathematically. Therefore, the analysis of certain generic nonlinear models might be interesting in a range of physical contexts. One of the important examples of such generic nonlinear models is the complex GinzburgLandau equation (CGLE), which was originally proposed as a phenomenological approach in the context of phase transitions [1], and is arguably one of the most studied nonlinear models in the physics community. It arises in physics, in particular, as a first-approximation "envelope" (or "amplitude") equation that governs the nonequilibrium dynamics of nonlinear systems in the presence of gain/loss and other effects such as linear and nonlinear dispersion or gain/loss saturation, depending on the specifics of the physical problem. It describes on a qualitative, and often quantitative level a vast variety of physical phenomena, from nonlinear waves to second-order phase transitions, superconductivity, superfluidity, Bose-Einstein condensation, liquid crystals, and strings in field theory [2]. In particular, equations of CGLE type have been used extensively to model mode-locked lasers [3-16]. The most well-known example in this context is referred to as the master equation of passive mode-locking [3-5]. This model belongs to the general class of so-called nonlinear dissipative systems, in which energy balance is provided by the interplay between external energy pumping and dissipative effects. An important and well known case of a conservative (energy preserving) limit of the general CGLE is the nonlinear Schrödinger equation (NLSE), which is widely used to describe high-frequency nonlinear wave propagation in a medium with Kerr-type nonlinearity when gain/loss effects do not make essential contributions to the nonlinear wave dynamics, or when their overall effect is averaged out leading to the pure NLSE. The latter is an 
example of an integrable Hamiltonian system [17, 18]. The NLSE is one of the very important underlying models in fibre optics. It was first proposed in the context of fibre optic communications [19-21], but has arguably even more important applications in all-optical signal processing, pulse shaping, optical data regeneration, frequency conversion, and the design and operation of a number of nonlinear photonic devices. Many of these applications are based on the existence of a robustly stable solution of the NLSE-the fundamental soliton.

The emergence of localized, particle-like structures that are called solitary waves or solitons is a widespread phenomenon occurring in a variety of physical problems. Classical optical solitons (i.e., solitons of the NLSE with uniform dispersion) are formed as a result of a continuous balance between the effects of linear dispersive pulse broadening and nonlinearity. An interesting modification of the classical soliton occurs in optical signal transmission along dispersion-managed (DM) links. In fibre optic communications, a periodic dispersion management-alternation of the fibre spans with positive and negative linear dispersionis an established technique to improve system performance and has been used to achieve ultra-long haul transmission systems [22]. Optical pulses propagating in a DM system experience a periodic change of their key parameters such as pulse width, power, and the phase parameter (chirp), following the periodic variations of the dispersion. Moreover, the use of periodic distributed Raman amplification in fibre lines makes it possible to control periodic variations of the signal power-the technique is known as nonlinearity management [23]. Averaging over the periodic variations of dispersion (and also over the nonlinear effects) leads to a new basic model that describes the slow dynamics of the optical pulses in such systems and a new breed of solitons [24]. The change in the sign of dispersion causes these socalled DM solitons to temporally broaden and recompress or "breathe" as they propagate. The prevalence of DM solitons has generated intensive study toward understanding their behaviour (see, e.g., [21, 22, 24-47]).

Recent developments in the field of nonlinear optics and optical communications are partially stimulated by the never-stopping demand of further increase of the capacity of fibre transmission systems. Efficient growth of the capacity of communication systems can be achieved by an increase in the channel bit rate- the speed at which information bits are transmitted. Increasing the channel rate assumes the utilization of shorter time slots allocated for each information bit and, consequently, of shorter carrier pulses. The propagation of ultrashort pulses is then strongly affected by the fibre dispersion, which results in large temporal broadening of the carrier pulses. Because of the temporal broadening during propagation, the carrier pulse power spreads over many time slots and so the accumulated effect of the instantaneous fibre nonlinearity tends to get averaged out. Signal transmission using very short optical pulses, often referred to as the quasilinear regime $[48,49]$, has become mainstream to the modern development in optical fibre communications. Traditional DM solitons typically have large energies for such short pulse widths or do not exist at all at high map strengths (the strength of the dispersion map will be defined later) [35]. In other terms, the Kerr nonlinearity cannot stabilize pulses in the form of traditional DM solitons at large strengths of the dispersion map. In this work we discuss two possible directions of further development of the DM soliton theory and corresponding applications. We consider two challenges that arise in the practical applications of solitons at large dispersion map strengths. On the one hand, in fibre optics, it is important to keep the energy of the carrier pulses below a certain level which is determined by the imposed power consumption and safety conditions. On the other hand, in powerful laser systems, the challenge is somewhat opposite- to generate stable optical pulses with as high energy as possible. Here we discuss both these physical problems by applying similar mathematical approaches. In fibre communications it would be of interest to find periodic solutions of the NLSE resembling traditional DM solitons, while having smaller energies. One possible solution to this problem was proposed in [50], and is known as short-scale or dense dispersion management [50-54]. Using dispersion management with a period that is much shorter compared to the length scale where nonlinear effects become significant, it is possible to reduce the energy of the DM soliton to levels that are acceptable for fibre transmission even when rather short pulses are used. This transmission technique can be important for channel rates of the scale of $100 \mathrm{Gbit} / \mathrm{s}$. We have recently shown that lower energy for the same pulse width can be achieved considering solutions of the NLSE with periods multiple to that one of the dispersion variation [55]. In this paper, we outline the main results of the analysis presented in [55]. We note that such multipleperiod solutions present somehow an important step in the convergence of the DM soliton concept and the widely used in practice quasilinear transmission regime. Namely, multiple-period DM solitons experience larger broadening during propagation and, therefore, much more closely mimic quasilinear propagation regimes compared to traditional DM solitons.

In the context of powerful laser systems, we analyze mode-locked lasers and study the properties of periodic (DM) solutions. The evolution of an optical pulse inside a laser cavity implemented either through a ring laser configuration or using a Fabry-Perot cavity design has an inherent periodicity related to the resonator round trip. Effects of gain and loss, dispersion, nonlinearity, gain dispersion (spectral filtering), and saturable absorption all can be mathematically treated as periodic variations of the system parameters. Solitary wave solutions of the CGLE for such systems are pulses that simultaneously balance the phases accumulated from dispersion and nonlinearity as well as the amplitude modulations from gain, linear and nonlinear (saturable) loss, and spectral filtering. Soliton solutions in a system with loss and gain are referred to as dissipative solitons $[56,57]$. Intuitively, such pulses balance two sets of modulations, compared to the lone balance of phase modulations in solitons of the NLSE. While conservative solitons have a continuum spectrum of their parameters, that is, they form families, dissipative solitons, as a result of the additional balance between gain and losses, usually do not form 
families, and their parameters are prescribed by properties of the system. The term "autosoliton" is also often used to stress the latter feature of such structures. Autosolitons can be viewed as attractors for the dynamical system or nonlinear "modes" of the system [56]. The first soliton fibre lasers have been constructed entirely of fibre with anomalous groupvelocity dispersion (GVD) to generate fundamental solitons of the NLSE [58]. However, the pulse energy is restricted by the soliton area theorem and sideband instabilities. Much higher energies are obtained when the laser has segments of normal and anomalous GVD, so the cavity consists of a dispersion map $[59,60]$. In general, the pulse breathes as it traverses the cavity, similar to the pulse dynamics in fibre communication systems with dispersion management. DM solitons (often called stretched pulses in the context of laser applications) are observed as the net or path-averaged cavity dispersion varies from relatively small and anomalous to small and normal $[59,60]$, while self-similar [61] and wavebreaking-free [62] pulses are observed with larger normal GVD. Pulses propagating in solid-state or hybrid (solidstate and fibre) mode-locked lasers are also often dispersion managed in order to achieve short pulse generation directly from the cavity rather than relying on further compression. In addition, both fibre and solid-state lasers exhibit periodic variations of nonlinearity inside the cavity, thus the pulse undergoes a simultaneous nonlinearity management. In lasers with soliton-like pulse shaping dissipative processes play a crucial role in driving the system to the steady-state evolution. On the other hand, the dynamics of the steadystate solution is dominated by the competing mechanisms of Kerr nonlinearity and linear dispersion [4, 5, 63], with the dissipative processes often retreating to a secondary role. Understanding of the interplay between all relevant effects involved in pulse generation and stabilization holds the key to the design of advanced laser systems based on DM solitons with the required properties. In this paper, by means of extensive numerical simulations of the CGLE and a reduced variational model, we highlight how the different dissipative processes affect the main dynamics of nonlinear and DM pulses in a mode-locked laser.

\section{Dispersion-Managed Solitons}

The optical pulse propagation along a fibre link with varying dispersion is governed by the normalized NLSE (see, e.g., [27] for more details):

$$
i \frac{\partial u}{\partial z}+d(z) \frac{\partial^{2} u}{\partial t^{2}}+\sigma(z)|u|^{2} u=0 .
$$

In (1), $z$ is the propagation distance, scaled on the dispersion compensation length $L, t$ is the retarded time, measured in units of a characteristic pulse width $T_{0}$, and $u(z, t)$ is the envelope of the electric field, normalized by the pulse peak power $P_{0}$. Periodic functions $d(z)=-\beta_{2}(z) L /\left(2 T_{0}^{2}\right)$ $(d(z+1)=d(z))$ and $\sigma(z)=\gamma \exp \left(2 \int_{0}^{z} G\left(z^{\prime}\right) \mathrm{d} z^{\prime}\right)$ describe, respectively, the varying dispersion and the signal power variations due to fibre loss and either distributed or lumped optical amplification. Here, $\beta_{2}$ is the GVD coefficient, $\gamma=2 \pi n_{2} P_{0} L /\left(\lambda_{0} A_{\text {eff }}\right)$ is the (normalized) Kerr nonlinearity coefficient, where $n_{2}$ is the nonlinear refractive index, $\lambda_{0}$ is the carrier wavelength, and $A_{\text {eff }}$ is the effective fibre area. The function $G(z)$ is the effective gain/loss function along the fibre line. Note that other material properties can lead to a varying nonlinear coefficient, such as a varying fiber core diameter [47]. Here we will consider the case of constant $\sigma$, which corresponds to the lossless case. In fiber optic communications, this situation corresponds to the rather typical limit where the scale of the periodic variations of gain is much smaller than the dispersion compensation period $L$. However, the results presented in this paper are not specific for and not limited to $\sigma=$ constant. When we consider mode-locked fiber lasers, the gain/loss perturbations will be considered as perturbative terms in the right-hand side of (1). Here we consider a two-step map with piecewiseconstant $d(z)$ and $\sigma(z)$ :

$$
\begin{array}{llll}
d(z)=d_{1}+\bar{d}, & d_{1}>0, & \sigma=\sigma_{1}, & 0<z<l_{1}, \\
d(z)=d_{2}+\bar{d}, & d_{2}<0, & \sigma=\sigma_{2}, & l_{1}<z<1,
\end{array}
$$

where $\bar{d}$ is the path-averaged (over the map period) dispersion, and $l_{1}, l_{2}=1-l_{1}$ are the lengths of the two pieces of fibre.

The traditional DM soliton [21, 24-44] is a solution of (1) that restores its form periodically with the period $L$. It can be considered as a nonlinear Bloch wave function or, in other terms, as a nonlinear eigenfunction of (1) with the period $L$ that exists in the case of small nonlinearity [28]. Indeed, due to the rapidly varying local dispersion, the DM pulse dynamics can be averaged to give a nonlocal equation in the spectral domain describing the slow evolution of the pulse envelope, the so-called DM NLSE [24, 25]. This equation has stable DM soliton solutions of the form $\hat{U}(z, \omega)=\exp \left(i \lambda^{2} z / 2\right) \hat{f}(\omega)$, where $\hat{U}$ is a Fourier-like transform of the field $u, \lambda^{2}$ is a constant, and $\hat{f}(\omega)$ is real and symmetric [26]. When these solutions seed (1), they remain localized and stable while acquiring an additional phase term. This frequency-dependent phase introduces a chirp in the pulse with a period related to the periodicity of the dispersion map. As the DM soliton propagates from the center of the normal dispersion region, it temporally broadens and then recompresses until it reaches the center of the anomalous region, whereupon it begins to broaden again, thereby breathing, as seen in Figure 1. The traditional DM soliton solutions of (1) have been studied comprehensively in literature [21, 24-44].

In general, DM solitons are very stable structures that exist for a wide range of parameter space, in bound-state configurations [45], and in the presence of higher order dispersion mapping [46]. One of the important issues concerning traditional DM solitons that is relevant for this work is when variations of the normalized function $d(z)$ are large (i.e., at large dispersion map strengths), such solitons either do not exist (are unstable) or their energy is too large to be practically used [35]. In the following two sections, we consider new aspects to DM systems. To obtain an understanding of these systems, we rely on simplified models that approximates the periodic solutions of the NLSE/CGLE 


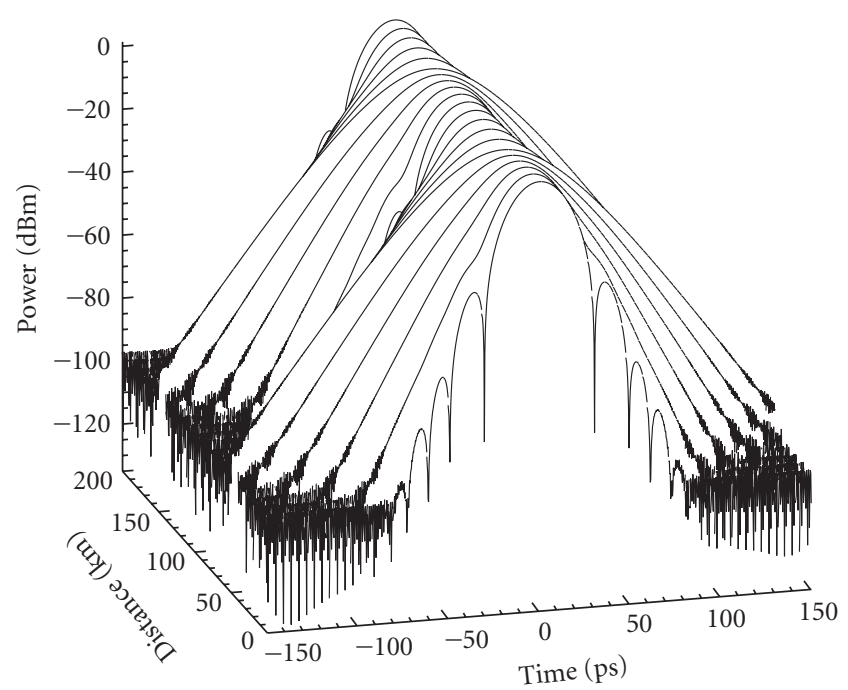

FIGURE 1: Breathing dynamics of a DM soliton $|u|^{2}$ (1) (on a logarithmic scale) propagating over one dispersion map period.

through a system ordinary differential equations (ODEs). Literature regarding variational reductions in nonlinear Schrödinger/Ginzburg-Landau type systems is vast, and has been used successfully to describe DM systems [36-44] as well as mode-locked lasers [64-67].

\section{Multiple-Period Dispersion-Managed Solitons}

In this section, we present a class of multiple-period solutions of the NLSE with varying dispersion [55]. While we examine general features of the NLSE rather than any specific application, without loss of generality we will use a terminology relevant to fibre optics applications.

Along with the standard solutions with period $L$ shown in the previous section, the NLSE (1) also possesses a class of eigenfunctions with multiple periods $L_{n}=n L$, where $n$ is a positive integer. A family of periodic solutions representing eigenfunctions of (1) with multiple periods has been presented in [55]. In general, such solutions have energy reduced compared to the corresponding standard DM solitons and, therefore, could be of a potential interest for high-speed transmission regimes. These multiple-period solutions can be analyzed by a simplified model that approximates the periodic solutions of the NLSE through a system of two ODEs. In what follows we will call the solutions with $n=1$ single-period solutions (or standard DM solitons), and those with $n>1$ multiple-period (or longperiod) solutions. The long-period solutions have not yet been studied systematically, and here we overview the results presented in [55].

3.1. Reduced Model: TM System. As mentioned before, in the limit when dispersion dominates nonlinearity, the pulse solution of the NLSE (1) experiences quasilinear oscillations during evolution, following the periodic variations of the dispersion. Effects of nonlinearity and small average dispersion come into play on much longer scales compared to $L$. The fast and slow dynamics of the NLSE solution can be separated by applying the substitution

$$
u(z, t)=N \frac{f(x, z)}{\sqrt{T(z)}} \exp \left[i \frac{M(z)}{T(z)} t^{2}\right]
$$

to (1), where $x=t / T(z)$ [37]. The parameter $N$ is introduced in (3) to scale out the pulse power by imposing a normalization condition on the structural (responsible for the pulse shape) function $f$ so that $\int|f|^{2} \mathrm{~d} x=1$. We define the evolution of $T$ and $M$ by (see, e.g., [27])

$$
\frac{\mathrm{d} T}{\mathrm{~d} z}=4 d(z) M, \quad \frac{\mathrm{d} M}{\mathrm{~d} z}=\frac{\mathrm{d}(z)}{T^{3}}-\frac{p \sigma(z) N^{2}}{T^{2}},
$$

where the parameter $p$ is constant. In what follows, we will refer to system (4) as the TM equations. Note that when $p=$ 0 system (4) describes the linear pulse evolution, and if $p \neq 0$ the additional term accounts in some sense for nonlinear evolution. Applying (4), we obtain the evolution equation for $f$ :

$$
i \frac{\partial f}{\partial z}+\frac{d(z)}{T^{2}}\left(\frac{\partial^{2} f}{\partial x^{2}}-x^{2} f\right)+N^{2} \frac{\sigma(z)}{T}\left(|f|^{2} f+p x^{2} f\right)=0 .
$$

For $p=0,(5)$ is the evolution equation of a quantum harmonic oscillator with an additional nonlinear term. The nonlinearity mixes different modes of the linear oscillator, inducing transitions from the ground state (which corresponds to a Gaussian-shaped pulse) to other modes. The constant $p$ in system (4) can be adjusted to minimize the rate of transitions from the ground state to other modes or, in other words, to make the approximation of the DM soliton solution of the NLSE as close as possible to the ground state of the linear operator. The intensity of the dispersioninduced pulse evolution can be characterized by the so-called map strength parameter $S$ defined (as in [35]) by

$$
S=2 \frac{l_{1} d_{1}-l_{2} d_{2}}{\min \left(\tau_{\mathrm{FWHM}}^{2}\right)}
$$

where $\min \left(\tau_{\mathrm{FWHM}}\right)$ is the minimum full-width at halfmaximum (FWHM) of the pulse taken along the dispersion map. It is known that for relatively large values of parameter $S$ the shape of the DM soliton is close to Gaussian [33]. In this case, the nonlinear term can be accounted for as an adiabatic perturbation to find the exact form of the pulse [27, 28]. Thus, under the assumption that the pulse shape is close to Gaussian, the problem of calculating the eigenfunctions of the NLSE (1) is reduced to the problem of calculating the periodic solutions of the TM system (4).

We note that system (4) can also be regarded as an approximate system of evolution equations for integral rootmean-square momenta of the pulse $[27,39]$. The function $T$ 


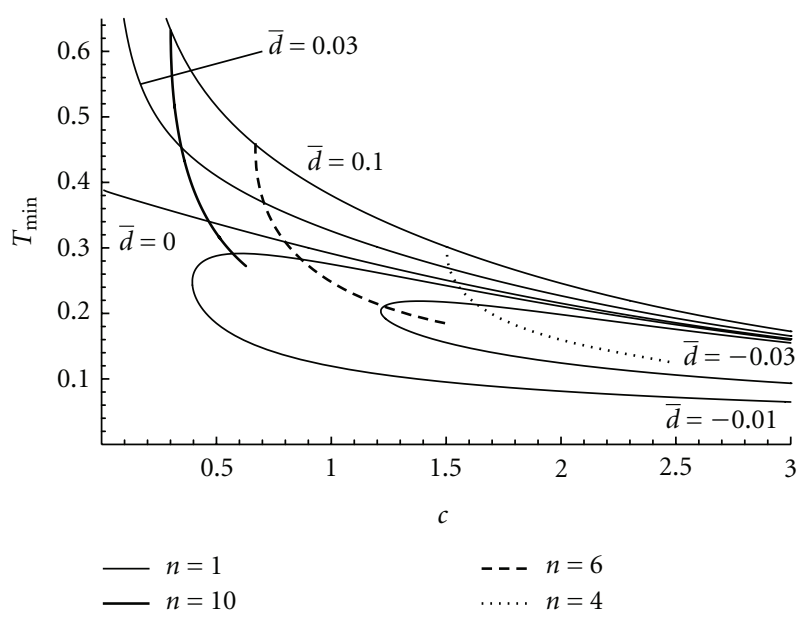

Figure 2: Dependence of minimum $T$ on the energy $c$. The thin lines correspond to single-period solutions $(n=1)$ with $\bar{d}=$ $0.1,0.03,0,-0.01,-0.03$ (from upper to lower line). The thick lines corresponds to multiple-period solutions with $\bar{d}=0.1$; the solid, long-dashed and short-dashed lines are for $n=10, n=6$, and $n=4$, respectively.

is related to $T_{\mathrm{int}}$ and $M$ corresponds to $M_{\mathrm{int}}$, where $T_{\mathrm{int}}$ and $M_{\text {int }}$ are defined as

$$
\begin{aligned}
T_{\text {int }} & =\sqrt{\frac{\int t^{2}|u|^{2} \mathrm{~d} t}{\int|u|^{2} \mathrm{~d} t}}, \\
T_{\text {int }} M_{\text {int }} & =\frac{i \int \frac{\int t\left(u u_{t}^{*}-u^{*} u_{t}\right) \mathrm{d} t}{4|u|^{2} \mathrm{~d} t} .}{}
\end{aligned}
$$

The evolution of $T_{\text {int }}$ and $M_{\text {int }}$ is governed by system (4) under the assumption that the phase of $u$ is quadratic in $t$. The accuracy of this assumption is the same as that of the assumption that the pulse shape remains Gaussian during propagation.

\subsection{Periodic Solutions of the TM Equations}

3.2.1. Revision of Single-Period Solutions. Without loss of generality, we consider the TM system (4) on a two-step map as defined by (2). Using a change of units of the variable $z$, we can set $d_{1}=1$ and $d_{2}=-1$. The map is then characterized by three parameters: the nonlinear coefficients of the two fibre segments $c_{1,2}=p \sigma_{1,2} N^{2}$ and the average dispersion $\bar{d}=l_{1}-l_{2}$. The map strength (6) takes the form $S=2 / \min \left(\tau_{\mathrm{FWHM}}^{2}\right)$. In this work, we only consider the case of "equally nonlinear" fibres, that is, $\left|c_{1} / d_{1}\right|=\left|c_{2} / d_{2}\right|=c$.

Here, we briefly recall the properties of the simple periodic solutions of the TM sytem. Let us consider the trajectory of one such solution in the $T-M$ plane. The condition that the solution is periodic can be written as

$$
z_{+}\left(T^{*}, M^{*}\right)=\frac{l_{1}}{2}, \quad z_{-}\left(T^{*}, M^{*}\right)=\frac{l_{2}}{2},
$$

where $\left\{T^{*}, M^{*}\right\}$ is the point of transition from anomalous fibre to normal fibre, and $z_{ \pm}(T, M)$ is the length of anomalous or normal fibre that takes the solution to pass from the point with $M=0$ to a given point $\{T, M\}$. This function can be found analytically $[28,29]$ to be

$$
z_{ \pm}=\frac{1}{2 V}\left[T M \mp \frac{c}{\sqrt{8 V}} \cosh ^{-1}\left(\frac{T \pm c /(2 V)}{\sqrt{c^{2} /\left(4 V^{2}\right)+1 /(2 V)}}\right)\right],
$$

where $V(z)=2 M^{2}+0.5 / T^{2} \mp c / T$. However, (8) provides a straightforward way of finding the single-period solutions of the TM system numerically. These solutions are comprehensively described in literature for some basic configurations [28]. Often the application of such solutions in the field of optical data transmission is directly related to the energy dependence of $T_{\min }=\min (T(z))$. This energy dependence is illustrated in Figure 2 (thin lines). As it should be, $T_{\min }$ tends to a finite value as $c \rightarrow 0$ in the case of zero $\bar{d}$, and tends to infinity when $\bar{d}>0$. For negative $\bar{d}$ the energy dependence of $T_{\min }$ has two branches.

3.2.2. Multiple-Period Solutions. To characterize multipleperiod solutions, it is convenient to define the transformation $g^{z}$ associated with the TM system which maps the $T-M$ plane onto itself. Then $g \equiv g^{1}$ is the mapping performed by one section of the fibre communication line. A simple periodic solution corresponds to a point $\left\{T^{*}, M^{*}\right\}$ mapped onto itself: $g^{1}\left(\left\{T^{*}, M^{*}\right\}\right)=\left\{T^{*}, M^{*}\right\}$. In other words, the point $\left\{T^{*}, M^{*}\right\}$ is a stable point of the map $g$. In the vicinity of this point, $g$ can be expanded in series with respect to $\delta T=T-T^{*}, \delta M=M-M^{*}$. In the area where this series converges, the motion under $g^{n}$ is regular, while outside of the area of convergence, the motion becomes chaotic. The trajectories in the regular area are closed curves encircling $\left\{T^{*}, M^{*}\right\}$. As it is typical for this mechanism of chaotization, the regular area is bordered by a separatrix, which separates the area where the orbits split into $n$ closed curves encircling some new central point $\left\{T_{m}^{*}, M_{m}^{*}\right\}$. The outmost $n$ curves form the separatrices, bordering this area on both inner and outer sides. Outside of it there is another area with closed orbits encircling $\left\{T^{*}, M^{*}\right\}$, then another area of the second type, and so on. The structure of the area separated by each of the $n$ outmost curves is the same as that of the big area. The $n$ points $\left\{T_{m}^{*}, M_{m}^{*}\right\}$ are (together with $\left\{T^{*}, M^{*}\right\}$ ) the stable points of $g^{n}$. The typical appearance of the trajectories is shown in Figure 3.

The solutions of the TM system corresponding to the stable points $\left\{T_{m}^{*}, M_{m}^{*}\right\}$ can be found numerically. Figure 4 shows an example of the evolution of $T(z)$ and $M(z)$ for a single-period DM soliton and a 4-period solution of the TM equations. The energy dependence of $T_{\min }$ for multipleperiod solutions with $n=10, n=6$, and $n=4$ at $\bar{d}=0.1$ is shown in Figure 2, and compared to the dependence of the simple periodic solution for the same map parameters.

It is customary to describe the properties of DM solitons in terms of map strength and normalized peak power $N_{\text {norm }}^{2}=\sigma P_{0} \tau_{\mathrm{FWHM}}^{2} /\left(3.11\left|\beta_{2}\right|\right)$ (see [35]). The dependence of the normalized peak power on the map strength for multipleperiod solutions is illustrated in Figure 5, and compared to the same dependence for single-period solutions. The multiple periodic lines branch off the lines corresponding 


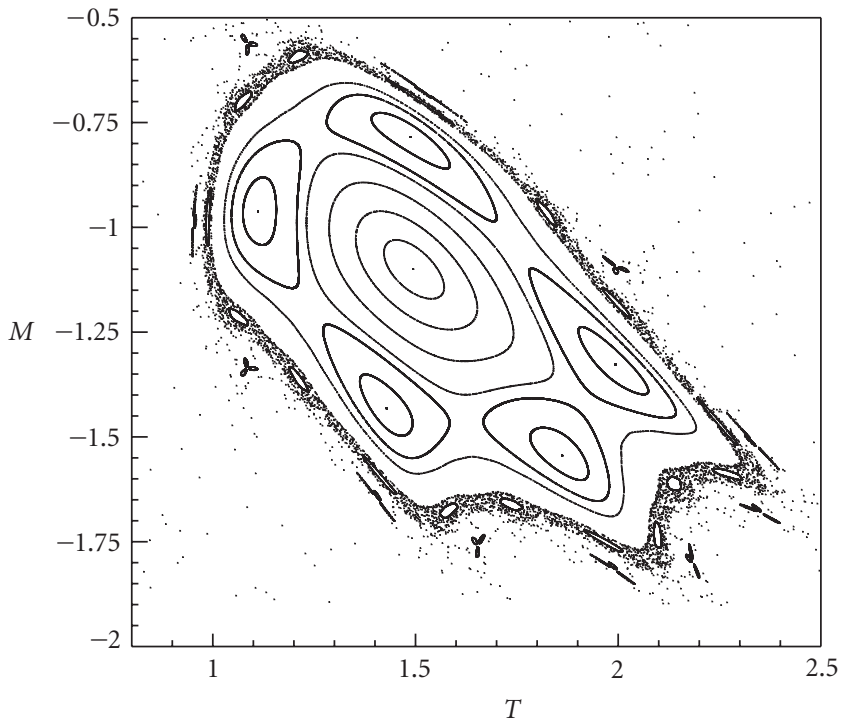

Figure 3: Trajectories of the points on the $T-M$ plane under $g^{n}$ for $\bar{d}=0$ and $c=3.5$.

to the simple periodic solutions on the same map. The line characterizing a multiple-period solution disappears when the corresponding point on the phase plane deepens into the chaotic area. As it can be seen from Figure 5, solutions with lower $n$ appear at higher energies. For zero average dispersion there is an $n$-period solution for every $n \geq 4$.

It is evident from the structure of the trajectories of the phase-plane points under $g^{n}$ (Figure 3 ) that in the vicinity of every stable point of $g^{n}$ there can exist stable points of $\left(g^{n}\right)^{m}$ for some $m$. These points could be called multiple-period solutions of the second order or $(n \times m)$ period solutions. For instance, the structure resembling a three-leaved flower in Figure 3 indicates the presence of solutions with $n=11$ and $m=3$. The lines representing such solutions on a $T_{\min }$ versus $c$ diagram must start from branches of their "mother" $n$ period solutions in the same way the $n$-period branches start from a single-period line. Undoubtdly, for large total periods solutions of higher orders can also be found, however, this is beyond the scope of the present work.

\section{Dispersion-Managed Solitons in Mode-Locked Fibre Lasers}

The multiple-period solutions of the previous section have less energy when compared with the single-period solutions of (1). This is advantageous in communication systems where low-energy pulses are required. In contrast, high-energy pulse solutions are preferred in fibre laser applications. Indeed, although the practical and innovative applications of mode-locked fibre lasers has continued to grow in the past decade [3], its broader impact has been limited due to restrictions on pulse energies, which is a consequence of the underlying cavity nonlinearities. To reduce the net nonlinear penalty, lasers have been constructed with dispersion maps with segments of alternating signs of
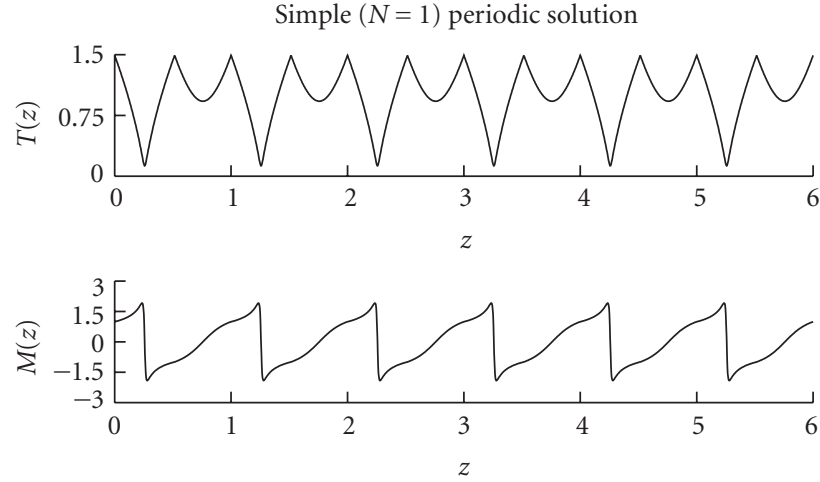

(a)
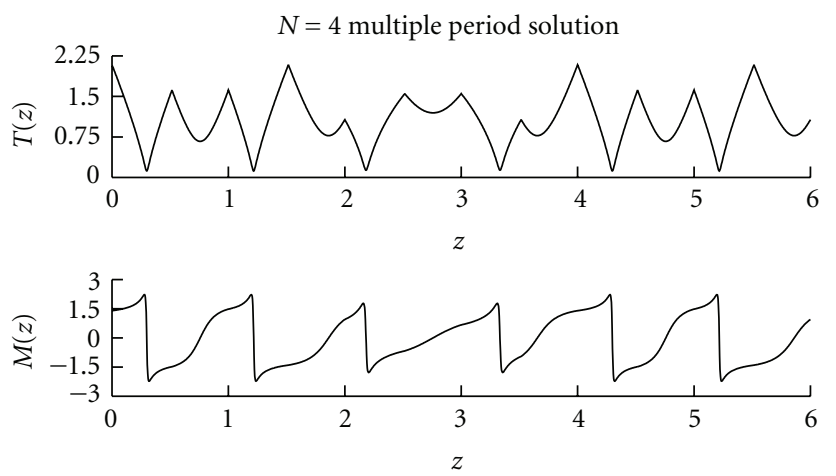

(b)

Figure 4: Evolution of $T$ and $M$ for a single-period DM soliton (upper plots) and a 4-period solution (lower plots). Here, $\bar{d}=0.03$ and $c=3.94$.

dispersion. As would be expected from linear propagation theory, the pulse stretches and compresses during each map period, effectively reducing the average pulse duration. Indeed, these so-called "stretched-pulse lasers" have resulted in femtosecond pulses with nanojoule energies and $\sim 1 \mathrm{~kW}$ power levels $[59,60]$. The evolution of the slowly varying electric field envelope in the laser cavity can be described at leading order by the NLSE (1) considered in the previous section [68]. Although the leading order terms consist of dispersion and self-phase modulation (SPM), certain dissipative terms are needed to achieve mode-locking. Here we analyze a modified DM system that includes these dissipative perturbations which are necessary for stable and robust mode-locking.

The evolution of electromagnetic energy in the laser cavity is subject to various dissipative effects such as attenuation, gain saturation, gain dispersion, and saturable absorption (intensity-discrimination). Haus proposed that these different elements could be averaged together resulting in the cubic-quintic Ginzburg-Landau evolution [3, 8-16]:

$$
\begin{aligned}
i \frac{\partial u}{\partial z} & +d(z) \frac{\partial^{2} u}{\partial t^{2}}+\gamma(z)|u|^{2} u \\
& =i\left(g(z)-l_{0}\right) u+i \tau g(z) \frac{\partial^{2} u}{\partial t^{2}}+i \beta|u|^{2} u-i \mu|u|^{4} u
\end{aligned}
$$




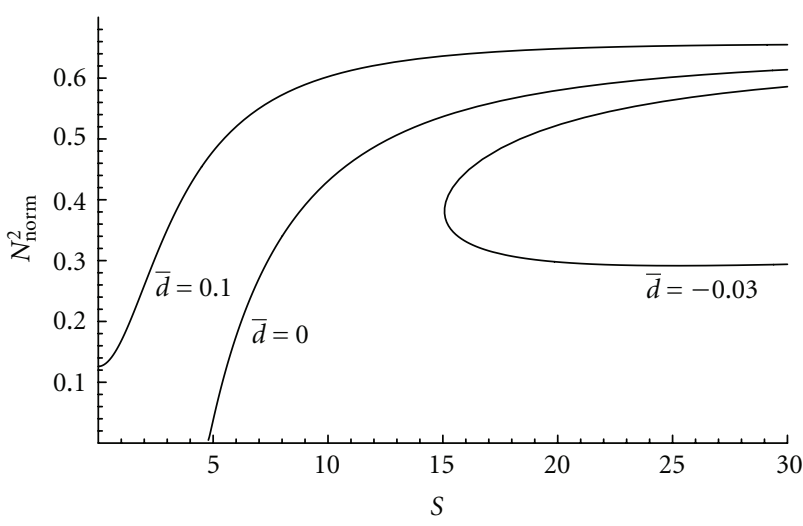

(a)

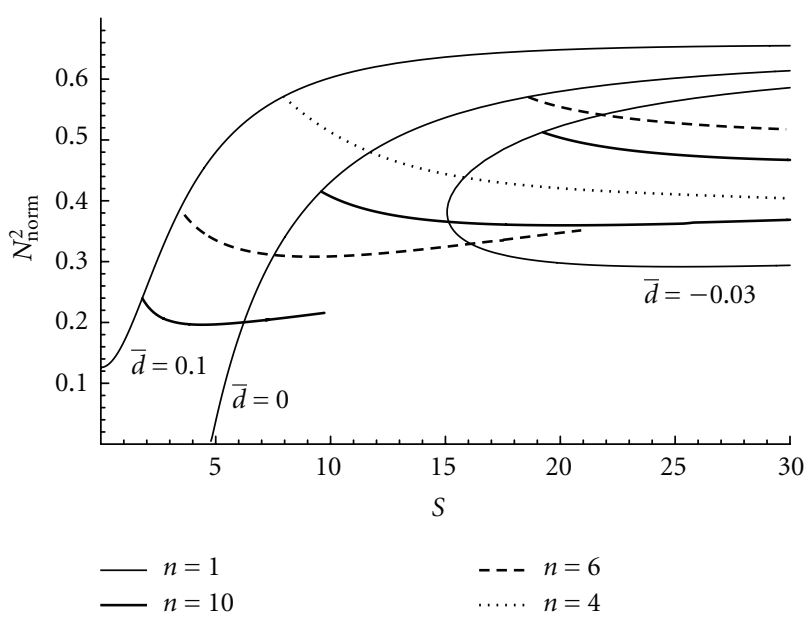

(b)

FIGURE 5: Dependence of the normalized power $N_{\text {norm }}^{2}$ on the map strength $S$. (a) shows simple periodic solutions with $\bar{d}=$ $0.1,0,-0.03$. The same simple periodic solutions are compared to multiple-period solutions (thick lines) in (b). The solid, longdashed, and short-dashed lines correspond to $n=10, n=6$, and $n=4$, respectively.

with saturated gain behavior given by [3]

$$
g(z)=\frac{g_{0}}{1+\|u\|^{2} / e_{0}} .
$$

In addition to the scalings for the standard NLSE (1), $g_{0}=$ $Z_{0} G, l_{0}=Z_{0} \Gamma, e_{0}=E_{\text {sat }} /\left(P_{0} T_{0}\right)$, and $\tau=1 /\left(\Omega_{g} T_{0}\right)^{2}$. Here the distance-scaling parameter $Z_{0}$ is the length of the laser cavity, $G$ (in $1 /$ meter) is the linear gain from amplification, $\Gamma$ (in $1 /$ meter) is the distributed losses in the cavity, $E_{\text {sat }}$ (in nanojoules) is the saturation energy of the gain medium, and $\Omega_{g}$ (1/second) is related to the gain bandwidth $\Delta \lambda$ through the relation $\Omega_{g}=\left(2 \pi c / \lambda_{0}^{2}\right) \Delta \lambda$. The self-amplitude modulation (SAM) parameters $\beta$ and $\mu$ provide a simple qualitative model for the intensity discrimination responsible for generating mode-locking. Only recently have these two key parameters been related to any physical process $[69,70]$, thus establishing asymptotic validity of the Haus model in a limited parameter regime. Regardless of this limited validity, the Haus model captures the key physical processes in the mode-locking physics and gives many important insights into the mode-locking dynamics.

The CGLE (10) is quite general and particular dynamics will depend on the parameter values. The parameter space for (10) is large, and is typically reduced by scaling the propagation distance by either the nonlinear or dispersion lengths. In the context of mode-locked lasers the dissipative terms play an important role in the scalings considered. The energy saturation determines the pulse power $P_{0}$, allowing us to choose the appropriate pulse duration $T_{0}$ to scale the ratio of the SPM coefficient $\gamma$ to the dispersion coefficient $d$ [71]. This scaling allows for a reduced parameter space to be considered. Here we are interested in how the various distributed dissipative terms present in a mode-locked fiber laser can change the nature of typical dispersion managed solutions.

4.1. Reduced Model. The governing equation (10) is a partial differential equation modeling the spatial-temporal evolution of electromagnetic energy in the laser cavity. To obtain a better understanding of the physical processes involved, a reduced system can be used to describe the complete evolution problem with ODEs that govern the evolution of a finite set of pulse parameters. Typically reduced systems are found in the context of classical Hamiltonian systems, however it is possible to use a modified variational $[67,72]$ or momentum [13,73] method which accounts for the dissipative terms in (10).

Inspired by numerical simulations and previous work done on DM solitons [27, 40,41], we use an ansatz of the form

$$
u(z, t)=\sqrt{\eta(z)} \exp \left[-(\omega(z) t)^{2}+i C(z) t^{2}+i \varphi(z)\right],
$$

where the intensity $(\eta)$, FWHM $(1 /(2 \omega))$, chirp parameter $(C)$, and phase-shift $(\varphi)$ all depend on propagation distance. Note that the dynamics of the pulse width $1 /(2 \omega)$ and chirp $C$ are easily related to the quantities $T$ and $M$ of the previous section. Using the modified variational method (for details see, e.g., $[67,72])$ with the ansatz $(12)$, the evolution of the ansatz parameters are found to satisfy the ODEs

$$
\begin{gathered}
\eta_{z}=-4 d C \eta+2\left(g-l_{0}\right) \eta+c_{1} \beta \eta^{2}-c_{2} \mu \eta^{3}-4 \tau g \eta \omega^{2} \\
\omega_{z}=-4 d C \omega+\frac{1}{2} \beta \eta \omega-c_{3} \mu \eta^{2} \omega-2 \tau g \omega\left[\omega^{2}-\left(\frac{C}{\omega}\right)^{2}\right] \\
C_{z}=4 d\left(\omega^{4}-C^{2}\right)-\frac{\sqrt{2}}{2} \gamma \eta \omega^{2}-8 \tau g C \omega^{2}
\end{gathered}
$$

where $c_{1}=5 \sqrt{2} / 3, c_{2}=16 /(3 \sqrt{3}), c_{3}=4 /(3 \sqrt{6})$, and the saturable gain is given by

$$
g=g(\eta, \omega)=\frac{g_{0}}{1+\sqrt{\pi / 2} \eta /\left(\omega e_{0}\right)} .
$$

This reduced model illuminates the dynamics in the laser cavity and can further our understanding of the dissipative processes in the context of dispersion and nonlinear management in mode-locked lasers. 


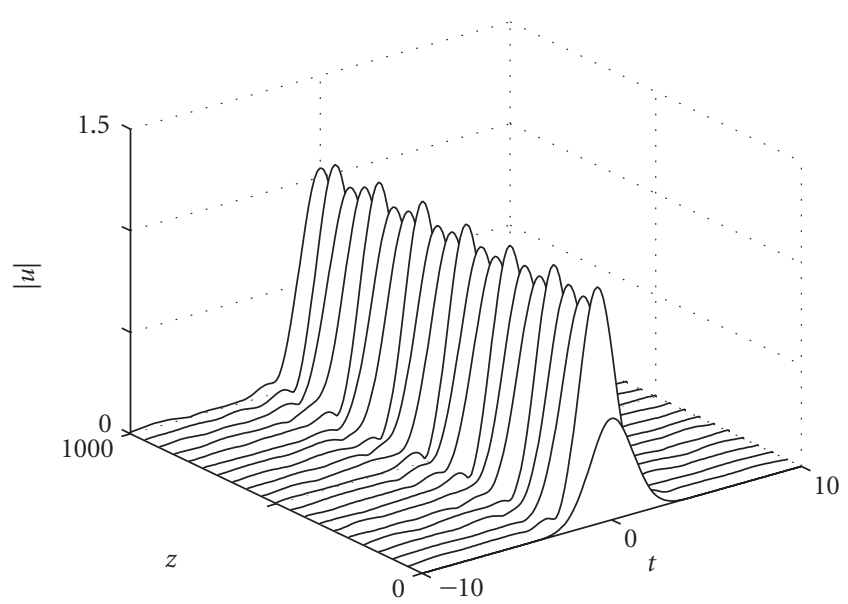

(a)

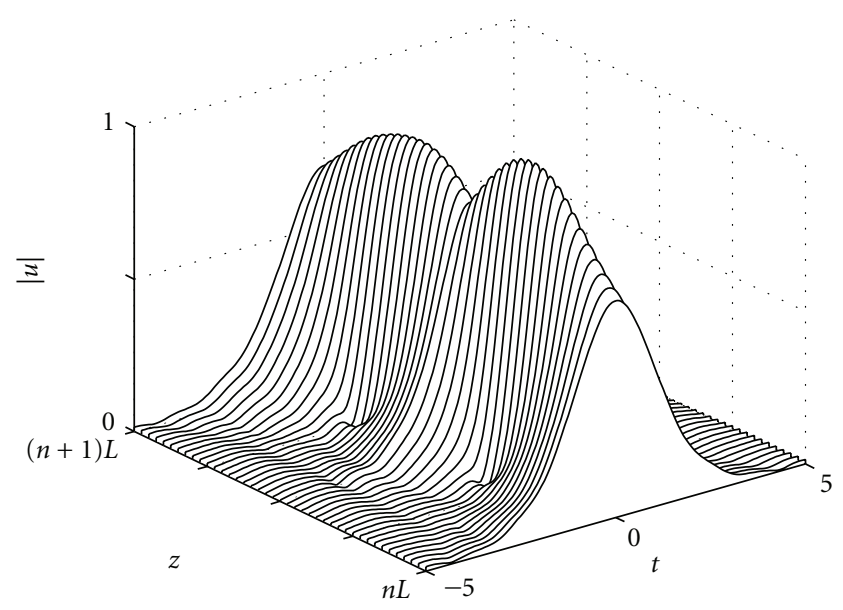

(b)

Figure 6: Pulse evolution obtained from solving the evolution equation (10) numerically for $\beta=\mu=\tau=0$. (a) Stroboscopic view where each pulse shown is located at the middle of the anomalous segment of the dispersion map. (b) Intramap evolution over one map period. Note that the intramap evolution is not exactly repeatable over consecutive map periods.

4.2. Numerical Simulations. To illustrate the mode-locking dynamics we consider a physically realizable two-step dispersion map (2) with the period $l=L / Z_{0}$ and where $d_{1}=$ $-d_{2}=d>0$. Further, as in the previous section, we restrict ourselves to the case of "equally nonlinear" fiber segments with $\gamma / d=1$. Note that a wide variety of dispersion maps can be considered. However, we have chosen this particular map to illustrate general trends in the behavior of dissipative DM mode-locking. For all simulations we use the dispersion map parameters $d=1, \bar{d}=0.1, l=0.5$, as well as gain saturation and loss parameters $g_{0}=2, l_{0}=1$, and $e_{0}=1$. In the following subsections, we will consider the case of infinite gain bandwidth and finite gain bandwidth separately.

4.2.1. Infinite Gain Bandwidth. To understand the effects gain saturation and SAM have on the governing equation

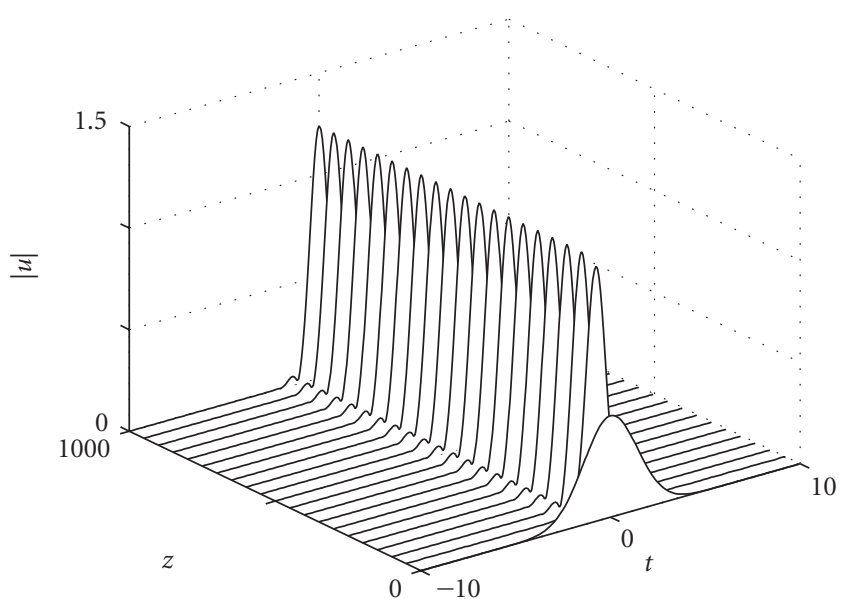

(a)

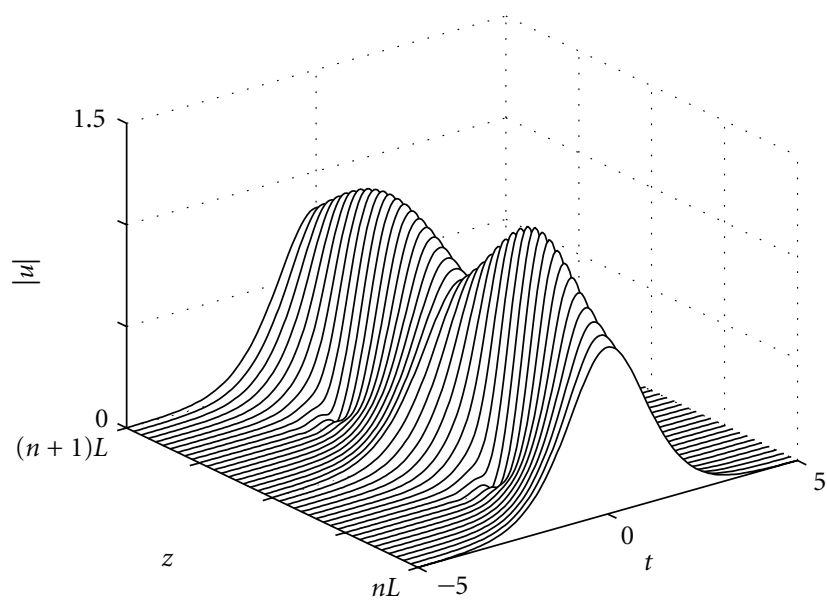

(b)

Figure 7: Pulse evolution obtained from solving the evolution equation (10) numerically for $\beta=0.2, \mu=0.05, \tau=0$. (a) Stroboscopic view where each pulse shown is located at the middle of the anomalous segment of the dispersion map. (b) Intramap evolution over one map period. Note that the intramap evolution is exactly repeatable over consecutive map periods.

(10), we consider the case where the gain bandwidth is much broader than the pulse bandwidth, that is, $\tau=0$. Figure 6 shows the result of simulation of (10) with only the effect of gain saturation included $(\beta=\mu=0)$. Here we see that the initial pulse quickly settles to a quasisteady state whose energy is given by $E=e_{0}\left(g_{0}-l_{0}\right) / l_{0}$. This is in contrast to DM solitons whose energy is determined by the energy of the input pulse. The gain saturation acts only as a selection mechanism, and once this quasisteady state is reached the evolution is completely determined by dispersion and SPM. Since the pulse configuration selected by the gain saturation typically does not coincide with what is needed for periodic pulse evolution for DM solitons, the intramap evolution in Figure 6 is not exactly repeatable over consecutive map periods.

Figure 7 shows the evolution of (10) with both gain saturation and SAM effects included $(\beta=0.2, \mu=0.05)$. 


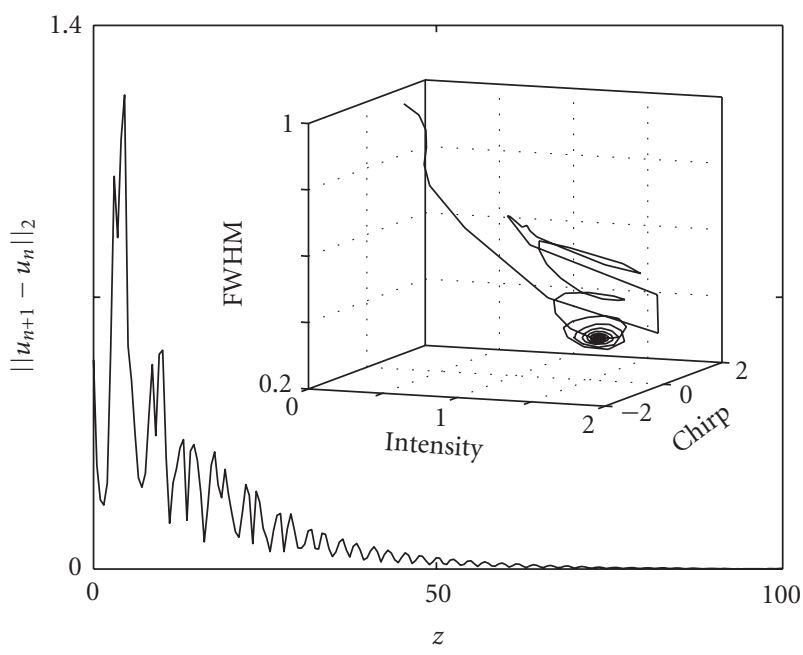

FIgURE 8: The two-norm of the difference between consecutive pulses which are located in the middle of the anomalous dispersion segment. Stable periodic evolution occurs as this quantity approaches zero. (Inset) Phase plane diagram showing the Poincaré section of consecutive pulses located in the middle of the anomalous dispersion segment.

Here we see that the combined effects creates a stable attracting periodic state. Figure 8 shows the two-norm of the difference between consecutive pulses which are located in the middle of the anomalous dispersion segment. It is clear that the evolution quickly settles from the initial condition towards a purely periodic state. The amplitude modulations from the saturable absorber eliminate any longscale dynamics. This is in contrast to DM systems where long-time oscillations occur [40]. The inset of Figure 8 shows the Poincare map for these pulses in a physically relevant phase space. Here the intensity, FWHM, and chirp parameter are calculated from numerical simulation using standard integral quantities-related $T_{\text {int }}$ and $M_{\text {int }}$ defined in (7). The phase line spirals into a point, showing the evolution goes to a purely periodic state.

A comparison between the solution to the reduced differential equations (13)-(15) and the full evolution equation (10) is shown in Figure 9 over one map period. Here the intensity $(\eta)$, FWHM $(1 /(2 \omega))$, and chirp parameter $(C)$ are shown for both cases. Although the reduced model is constrained by the ansatz assumption, it is remarkable how accurately it models the full equation dynamics. One slight difference is that the reduced model solution parameters experience long-scale oscillations which are not present in the full solution to (10). However, these oscillations cause a maximum error of $\sim 7$ percent. Figure 10 shows the phase plane dynamics over 100 periods for both the reduced model (dashed curves) and the full evolution equation (grey curve). Here we see the typical "crescent moon" structure that is observed in DM soliton theory [27, 40,41]. Note that the long-scale oscillations observed in the reduced model is visible due to the quasi-periodic orbits. Although there is slight variations, the reduced set of ODEs captures the essential dynamics of the full evolution equation.

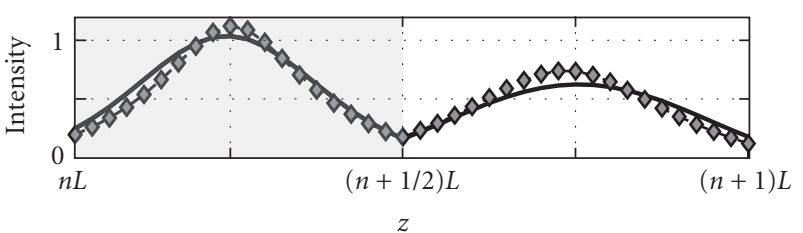

(a)

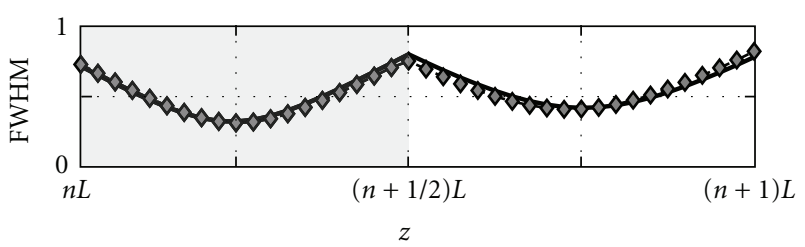

(b)

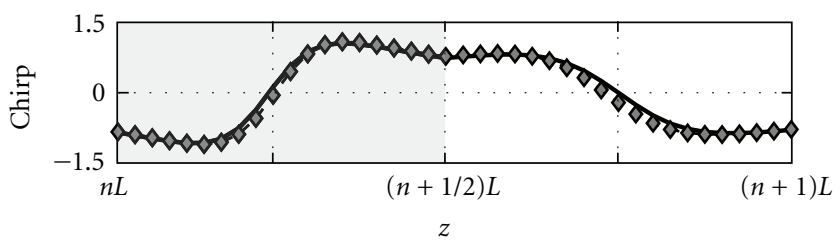

(c)

FIGURE 9: Evolution of the intensity, FWHM, and chirp parameter over one map period in the case where only gain saturation and SAM are present. The results from both the full evolution equation (10) (solid black curves) and the reduced equations (13)-(15) (grey diamonds). The shaded section corresponds to the anomalous dispersion segment.

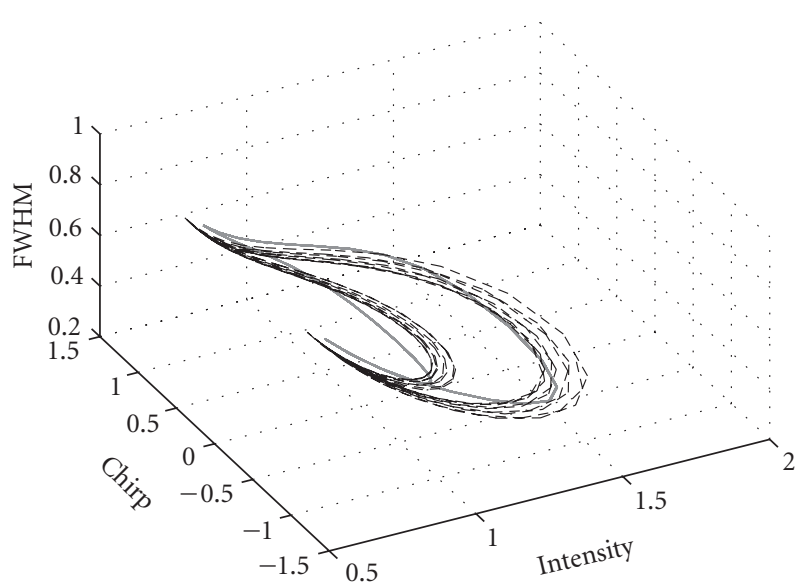

FIGURe 10: Phase plane dynamics over 100 periods after the pulse has reached steady-state for both the reduced model (dashed curves) and the full evolution equation (solid grey curve).

Although the amplitude modulations from the gain saturation and saturable absorber create a stable attracting state, the overall structure of the steady state is similar to the DM soliton. Specifically, at the center of each segment in the map there exists a peak-amplitude and minimum pulsewidth corresponding to the zero-chirp points. Further, the side-lobe structure observed for DM solitons at maximum compression exists as well. Thus gain saturation along with 


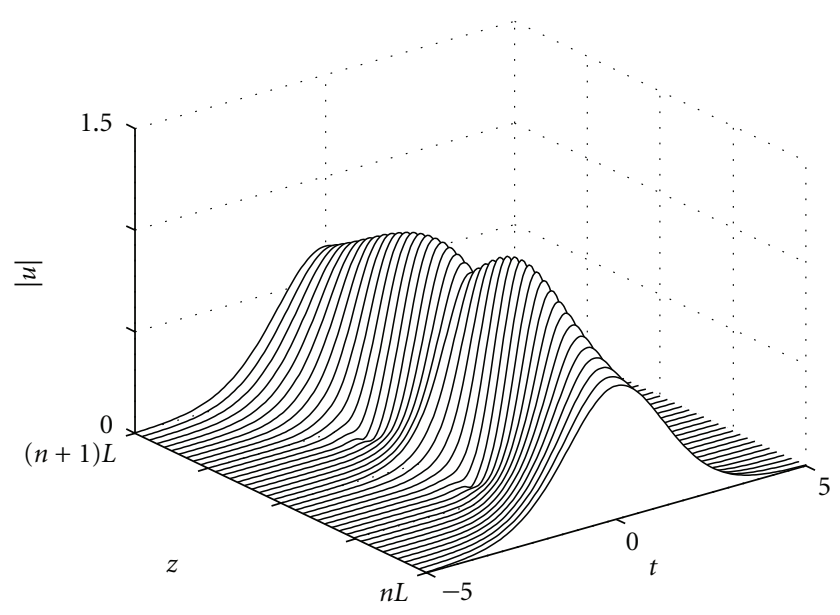

(a)
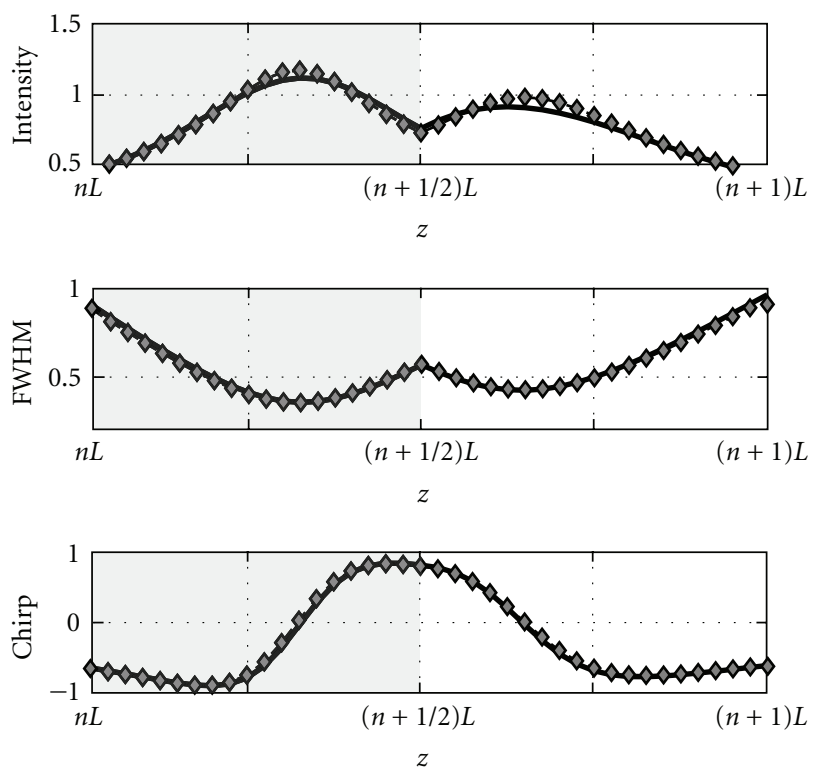

(b)

Figure 11: One-map period evolution in the case where gain saturation, saturable absorber, and gain bandwidth are included ( $\beta=0.2, \mu=\tau=0.05$ ). (a) Intramap evolution from numerical solution of (10). (b) Evolution of the intensity, FWHM, and chirp parameter over one map period from both the full evolution equation (10) (solid black curves) and the reduced equations (13)(15) (grey diamonds). The shaded section corresponds to the anomalous dispersion segment.

SAM selects the exact dissipative DM soliton that will satisfy the energy constraints. In general, gain saturation and SAM allow for high map-strength evolution to persist resulting in higher-energy pulses than those considered in the previous section.

4.2.2. Finite Gain Bandwidth. When the pulse bandwidth is comparable to the gain bandwidth, we must include the effects of bandwidth-limited gain. For a gain bandwidth which can vary from $\Delta \lambda=20-40 \mathrm{~nm}, \tau \approx 0.03-0.32$.

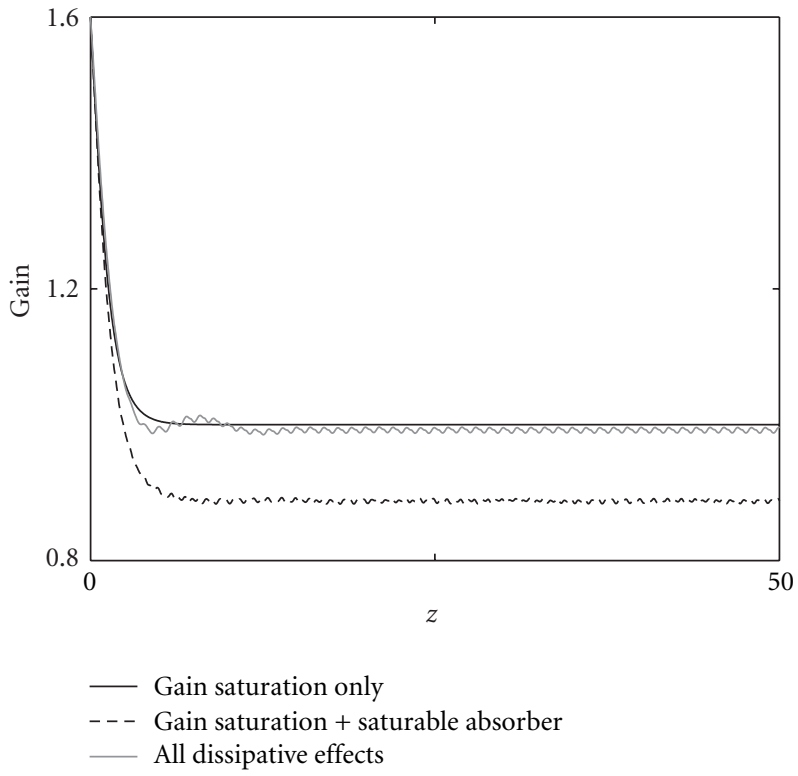

FIGURE 12: Numerically calculated gain from three different simulations of (10) which include the dissipative effects: gain saturation; gain saturation and SAM; gain saturation, SAM and bandwidth limited gain. Note that the gain saturates to a constant value in all cases.

In general, including the gain-bandwidth term does not destabilize the periodic evolution. However, it does change the nature of the periodicity. Figure 11 shows the intraperiod evolution as well as the intensity, FWHM, and chirp parameter from both the full simulated equation (10) and the reduced system (13) $-(15)$. Here the equation parameters are taken to be $\beta=0.2, \mu=\tau=0.05$. From this simulation we see that the steady-state periodic solution persists, as well as typical DM soliton structure such as side-lobes. However, the zero-chirp (minimum FWHM) points are much closer to the center point of the map. Indeed, we see that although the gain saturation and SAM have no contribution to (15), the gain bandwidth clearly does. As $\tau$ is increased the zerochirp points coincide and the structure loses stability. Again it should be noted that the reduced model (13)-(15) does an excellent job characterizing the dynamics of the full evolution equation (10).

4.3. Steady-State Dynamics. The dissipative perturbations considered selects the exact DM soliton that will satisfy particular energy constraints. Figure 12 illustrates that the gain (energy) nearly saturates to a constant value in all cases we have considered. Thus, once mode-locking has reached its steady-state, $d E / d z \sim 0$. We can use this conserved quantity in the reduced model (13)-(15) to lower the dimensionality of the system. At steady-state, $\eta_{z} \omega-\eta \omega_{z}=0$, giving

$$
2\left(g-l_{0}\right) \sim b_{1} \beta \eta+b_{2} \mu \eta^{2}+2 \tau g\left[\omega^{2}+\left(\frac{C}{\omega}\right)^{2}\right],
$$


where $b_{1}=1 / 2-c_{1}$ and $b_{2}=c_{2}-c_{3}$. Using (17) in (13) we see that $\eta=\alpha \omega$ for some constant $\alpha$. This gives the twodimensional steady-state system

$$
\begin{gathered}
\omega_{z}=-4 d C \omega+\frac{\alpha}{2} \beta \omega^{2}-c_{3} \alpha^{2} \mu \omega^{3}-2 \tau g \omega\left[\omega^{2}-\left(\frac{C}{\omega}\right)^{2}\right], \\
C_{z}=4 d\left(\omega^{4}-C^{2}\right)-\frac{\sqrt{2} \alpha}{2} \gamma \omega^{3}-8 \tau g C \omega^{2} .
\end{gathered}
$$

These coupled equations govern the dynamics of the pulse once the transient behavior has died out and the energy has saturated to a (nearly) constant value.

Since the reduced system provides an excellent description of the full model, it can be used to calculate relevant physical quantities. For example, the chirp parameter $C_{0}$ at minimum FWHM $\omega_{0}$ is found by setting (18) to zero. For the case of infinite bandwidth we have

$$
C_{0}=\frac{\alpha \omega_{0}}{4 d}\left[\frac{\beta}{2}-\alpha c_{3} \mu \omega_{0}\right]
$$

In the case of a finite gain bandwidth, we have

$$
\begin{aligned}
C_{0} & =\frac{d \omega_{0}^{2}}{2 \tau g}\left[1 \pm \sqrt{1-\frac{\tau g}{2 d^{2}}\left(\frac{\alpha \beta}{2 \omega_{0}}-\alpha^{2} c_{3} \mu-2 \tau g\right)}\right] \\
& \sim \frac{\alpha \omega_{0}}{2 d}\left[\frac{\beta}{4}-\left(\frac{\alpha c_{3} \mu}{2}-\tau g\right) \omega_{0}\right],
\end{aligned}
$$

where we obtain the approximation (22) by taking the minus sign in (21) and using the fact that the dissipative parameters $\beta, \mu$, and $\tau$ are much less than unity. Note that since these parameters are small, the minimum pulse duration occurs when $\left|C_{0}\right| \ll 1$. Further, it is interesting to note that value of $C_{0}$ is inversely proportional to the map depth $d$. Thus for large map depths, the minimum FWHM of the pulse will occur when $C \sim 0$, which is consistent for dispersion dominated systems.

It should be noted that all Hamiltonian terms (with $\alpha=$ 1) in (18)-(19) were obtained in the context of DM solitons in $[40,41]$. There, the typical "crescent moon" structure was observed and related to stable DM propagation. In that work, the initial condition was critical in obtaining a closed loop in the phase space. Here the dissipative terms determine the energy of the pulse, and act as a stabilization mechanism to maintain a purely periodic solution. Further, they allow for large map strength evolution resulting in high-energy pulse propagation.

\section{Conclusion}

We have discussed some recent developments in the theory of DM solitons within the context of photonic applications. We have considered two directions of further development of the DM soliton theory and corresponding practical applications in optical signal transmission/processing and mode-locked laser systems. In the context of optical communications, it is desirable to keep the energy of the carrier pulses below a certain level, which is imposed by power consumption and safety conditions. In the framework of a reduced ODE-based model, we have examined a class of periodic localized solutions of the NLSE with periodic variations of the dispersion. Such solutions have multiple periods and lower energies compared to traditional DM solitons of the same temporal width. The multiple-period DM solitons described in this work experience larger broadening during propagation and, therefore, they much more closely mimic the widely used in practice quasilinear transmission regime compared to traditional DM solitons.

In the context of powerful laser systems, we have examined mode-locked lasers and studied the properties of periodic dissipative DM solitons with high energies. Note that in powerful laser systems, one of the important goals is to generate stable optical pulses with as high energy as possible. By means of numerical simulations and a reduced variational model of the governing CGLE, we have analyzed the influence of the different dissipative processes that take place in the laser cavity. The reduced model introduced here rather accurately describes the key characteristics of the dissipative DM solitons, such as pulse width, chirp, peak power and energy. This model might be very useful for multiparametric optimization of complex laser systems.

\section{References}

[1] V. L. Ginzburg and L. D. Landau, "To the theory of superconductivity," Soviet Physics Journal of Experimental and Theoretical Physics, vol. 20, pp. 1064-1082, 1950.

[2] I. S. Aranson and L. Kramer, "The world of the complex Ginzburg-Landau equation," Reviews of Modern Physics, vol. 74, no. 1, pp. 99-143, 2002.

[3] H. A. Haus, "Mode-locking of lasers," IEEE Journal on Selected Topics in Quantum Electronics, vol. 6, no. 6, pp. 1173-1185, 2000.

[4] H. A. Haus, J. G. Fujimoto, and E. P. Ippen, "Analytic theory of additive pulse and Kerr lens mode locking," IEEE Journal of Quantum Electronics, vol. 28, no. 10, pp. 2086-2096, 1992.

[5] J. N. Kutz, "Mode-locked soliton lasers," SIAM Review, vol. 48, no. 4, pp. 629-678, 2006.

[6] J. Lega, J. V. Moloney, and A. C. Newell, "Swift-Hohenberg equation for lasers," Physical Review Letters, vol. 73, no. 22, pp. 2978-2981, 1994.

[7] G.-L. Oppo, G. D’Alessandro, and W. J. Firth, “Spatiotemporal instabilities of lasers in models reduced via center manifold techniques," Physical Review A, vol. 44, no. 7, pp. 4712-4720, 1991.

[8] N. N. Akhmediev and A. Ankiewicz, Eds., Solitons, Nonlinear Pulses and Beams, Chapman and Hall, London, UK, 1997.

[9] J. M. Soto-Crespo, N. N. Akhmediev, and V. V. Afanasjev, "Stability of the pulselike solutions of the quintic complex Ginzburg-Landau equation," Journal of the Optical Society of America B, vol. 13, no. 7, pp. 1439-1449, 1996.

[10] J. M. Soto-Crespo, N. N. Akhmediev, V. V. Afanasjev, and S. Wabnitz, "Pulse solutions of the cubic-quintic complex Ginzburg-Landau equation in the case of normal dispersion," Physical Review E, vol. 55, no. 4, pp. 4783-4796, 1997. 
[11] N. Akhmediev, J. M. Soto-Crespo, and G. Town, "Pulsating solitons, chaotic solitons, period doubling, and pulse coexistence in mode-locked lasers: complex Ginzburg-Landau equation approach," Physical Review E, vol. 63, no. 5, Article ID 056602, 4 pages, 2001.

[12] J. M. Soto-Crespo and N. Akhmediev, "Soliton as strange attractor: nonlinear synchronization and chaos," Physical Review Letters, vol. 95, no. 2, Article ID 024101, 4 pages, 2005.

[13] A. Ankiewicz, N. Devine, N. N. Akhmediev, and J. M. Soto-Crespo, "Dissipative solitons and anti-solitons," Physical Review A, vol. 370, pp. 454-458, 2007.

[14] N. Akhmediev, J. M. Soto-Crespo, and P. Grelu, "Roadmap to ultra-short record high-energy pulses out of laser oscillators," Physics Letters A, vol. 372, no. 17, pp. 3124-3128, 2008.

[15] E. Podivilov and V. L. Kalashnikov, "Heavily-chirped solitary pulses in the normal dispersion region: new solutions of the cubic-quintic complex Ginzburg-Landau equation," JETP Letters, vol. 82, no. 8, pp. 467-471, 2005.

[16] V. L. Kalashnikov, E. Podivilov, A. Chernykh, and A. Apolonski, "Chirped-pulse oscillators: theory and experiment," Applied Physics B, vol. 83, no. 4, pp. 503-510, 2006.

[17] V. E. Zakharov and A. B. Shabat, "Exact theory of two-dimensional self-focusing and onedimensional selfmodulation of waves in nonlinear media," Zhurnal Eksperimentalnoi i Teoreticheskoi Fiziki, vol. 61, pp. 118-134, 1971.

[18] V. E. Zakharov and A. B. Shabat, "Exact theory of two-dimensional self-focusing and onedimensional selfmodulation of waves in nonlinear media," Soviet Physics Journal of Experimental and Theoretical Physics, vol. 34, pp. 6269, 1972.

[19] A. Hasegawa and F. Tappert, "Transmission of stationary nonlinear optical pulses in dispersive dielectric fibers. I. Anomalous dispersion," Applied Physics Letters, vol. 23, no. 3, pp. 142-144, 1973.

[20] A. Hasegawa and Y. Kodama, Solitons in Optical Communications, Oxford University Press, Oxford, UK, 1995.

[21] L. F. Mollenauer and J. P. Gordon, Solitons in Optical Fibers: Fundamentals and Applications, Academic Press, 2006.

[22] L. F. Mollenauer, P. V. Mamyshev, J. Gripp, et al., "Experimental demonstration of massive WDM overtransoceanic distances using dispersion managed solitons," in Massive WDM and TDM Soliton Transmission Systems, A. Hasegawa, Ed., vol. 6, pp. 115-127, Springer, Dordrecht, The Netherlands, 2000.

[23] I. Nasieva, J. D. Ania-Castañón, and S. K. Turitsyn, "Nonlinearity management in fibre links with distributed amplification," Electronics Letters, vol. 39, no. 11, pp. 856-857, 2003.

[24] I. R. Gabitov and S. K. Turitsyn, "Averaged pulse dynamics in a cascaded transmission system with passive dispersion compensation," Optics Letters, vol. 21, no. 5, pp. 327-329, 1996.

[25] M. J. Ablowitz and G. Biondini, "Multiscale pulse dynamics in communication systems with strong dispersion management," Optics Letters, vol. 23, no. 21, pp. 1668-1670, 1998.

[26] Y. Chen and H. A. Haus, "Dispersion-managed solitons in the net positive dispersion regime," Journal of the Optical Society of America B, vol. 16, no. 1, pp. 24-30, 1999.

[27] S. K. Turitsyn, E. G. Shapiro, S. B. Medvedev, M. P. Fedoruk, and V. K. Mezentsev, "Physics and mathematics of dispersionmanaged optical solitons," Comptes Rendus Physique, vol. 4, no. 1, pp. 145-161, 2003.

[28] S. K. Turitsyn, T. Schäfer, K. H. Spatschek, and V. K. Mezentsev, "Path-averaged chirped optical soliton in dispersionmanaged fiber communication lines," Optics Communications, vol. 163, no. 1, pp. 122-158, 1999.
[29] T. I. Lakoba, J. Yang, D. J. Kaup, and B. A. Malomed, "Conditions for stationary pulse propagation in the strong dispersion management regime," Optics Communications, vol. 149, no. 4-6, pp. 366-375, 1998.

[30] I. Gabitov, E. G. Shapiro, and S. K. Turitsyn, "Asymptotic breathing pulse in optical transmission systems with dispersion compensation," Physical Review E, vol. 55, no. 3, pp. 3624-3633, 1997.

[31] T.-S. Yang and W. L. Kath, "Analysis of enhanced-power solitons in dispersion-managed optical fibers," Optics Letters, vol. 22, no. 13, pp. 985-987, 1997.

[32] I. Gabitov, E. G. Shapiro, and S. K. Turitsyn, "Optical pulse dynamics in fiber links with dispersion compensation," Optics Communications, vol. 134, no. 1-6, pp. 317-329, 1997.

[33] N. J. Smith, F. M. Knox, N. J. Doran, K. J. Blow, and I. Bennion, "Enhanced power solitons in optical fibres with periodic dispersion management," Electronics Letters, vol. 32, no. 1, pp. 54-55, 1996.

[34] N. J. Smith, N. J. Doran, F. M. Knox, and W. Forysiak, "Energyscaling characteristics of solitons in strongly dispersionmanaged fibers," Optics Letters, vol. 21, no. 24, pp. 1981-1983, 1996.

[35] A. Berntson, N. J. Doran, W. Forysiak, and J. H. B. Nijhof, "Power dependence of dispersion-managed solitons for anomalous, zero, and normal path-average dispersion," Optics Letters, vol. 23, no. 12, pp. 900-902, 1998.

[36] Y. Kodama, S. Kumar, and A. Maruta, "Chirped nonlinear pulse propagation in a dispersion-compensated system," Optics Letters, vol. 22, no. 22, pp. 1689-1691, 1997.

[37] E. G. Shapiro and S. K. Turitsyn, "Theory of guiding-center breathing soliton propagation in optical communication systems with strong dispersion management," Optics Letters, vol. 22, no. 20, pp. 1544-1546, 1997.

[38] S. K. Turitsyn, T. Schäfer, and V. K. Mezentsev, "Selfsimilar core and oscillatory tails of a path-averaged chirped dispersion-managed optical pulse," Optics Letters, vol. 23, no. 17, pp. 1351-1353, 1998.

[39] S. K. Turitsyn, T. Schäfer, and V. K. Mezentsev, "Generalized momentum method to describe high-frequency solitary wave propagation in systems with varying dispersion," Physical Review E, vol. 58, no. 5, pp. R5264-R5267, 1998.

[40] J. N. Kutz, P. Holmes, S. G. Evangelides Jr., and J. P. Gordon, "Hamiltonian dynamics of dispersion-managed breathers," Journal of the Optical Society of America B, vol. 15, no. 1, pp. 87-96, 1998.

[41] J. N. Kutz and S. G. Evangelides Jr., "Dispersion-managed breathers with average normal dispersion," Optics Letters, vol. 23, no. 9, pp. 685-687, 1998.

[42] A. V. Mikhailov, "Variationalism and empiriocriticism (Exact and variational approaches to fibre optics equations)," in Optical Solitons: Theoretical Challenges and Industrial Perspectives, V. E. Zakharov and S. Wabnitz, Eds., pp. 63-72, Springer, Berlin, Germany, 1999.

[43] B. A. Malomed, "Variational methods in nonlinear fiber optics and related fields," Progress in Optics, vol. 43, pp. 69-191, 2002.

[44] J. P. Gordon and L. F. Mollenauer, "Scheme for the characterization of dispersion-managed solitons," Optics Letters, vol. 24, no. 4, pp. 223-225, 1999.

[45] I. Gabitov, R. Indik, L. Mollenauer, M. Shkarayev, M. Stepanov, and P. M. Lushnikov, "Twin families of bisolitons in dispersion-managed systems," Optics Letters, vol. 32, no. 6, pp. 605-607, 2007. 
[46] J. Moeser, I. Gabitov, and C. K. R. T. Jones, "Pulse stabilization by high-order dispersion management," Optics Letters, vol. 27, no. 24, pp. 2206-2208, 2002.

[47] I. R. Gabitov and P. M. Lushnikov, "Nonlinearity management in a dispersion-managed system," Optics Letters, vol. 27, no. 2, pp. 113-115, 2002.

[48] R.-J. Essiambre, B. Mikkelsen, and G. Raybon, "Intra-channel cross-phase modulation and four-wave mixing in high-speed TDM systems," Electronics Letters, vol. 35, no. 18, pp. 15761578, 1999.

[49] P. V. Mamyshev and N. A. Mamysheva, "Pulse-overlapped dispersion-managed data transmission and intrachannel fourwave mixing," Optics Letters, vol. 24, no. 21, pp. 1454-1456, 1999.

[50] S. K. Turitsyn, M. P. Fedoruk, and A. Gornakova, "Reducedpower optical solitons in fiber lines with short-scale dispersion management," Optics Letters, vol. 24, no. 13, pp. 869-871, 1999.

[51] S. K. Turitsyn, N. J. Doran, E. G. Turitsyna, E. G. Shapiro, M. P. Fedoruk, and S. B. Medvedev, "Dispersion-managed transmission systems with short-scale dispersion management," in Massive WDM and TDM Soliton Transmission Systems, M. Shinomiya and A. Hasegawa, Eds., pp. 235-251, Kluwer Academic Publishers, Dordrecht, The Netherlands, 2000.

[52] A. H. Liang, H. Toda, and A. Hasegawa, "High-speed soliton transmission in dense periodic fibers," Optics Letters, vol. 24, no. 12, pp. 799-801, 1999.

[53] L. J. Richardson, W. Forysiak, and N. J. Doran, "Dispersionmanaged soliton propagation in short-period dispersion maps," Optics Letters, vol. 25, no. 14, pp. 1010-1012, 2000.

[54] M. Shtaif, "Ultrahigh data-rate transmission using a dense dispersion map with two-fold periodicity," IEEE Photonics Technology Letters, vol. 20, no. 8, pp. 620-622, 2008.

[55] O. Y. Schwartz and S. K. Turitsyn, "Multiple-period dispersion-managed solitons," Physical Review A, vol. 76, no. 4, Article ID 043819, 7 pages, 2007.

[56] N. Akhmediev and A. Ankiewicz, Eds., Dissipative Solitons, vol. 661 of Lecture Notes in Physics, Springer, Dordrecht, The Netherlands, 2005.

[57] Y. S. Kivshar and G. P. Agarwal, Optical Solitons: From Fibers to Photonic Crystals, Academic Press, 2003.

[58] L. F. Mollenauer and R. H. Stolen, "The soliton laser," Optics Letters, vol. 9, no. 1, pp. 13-15, 1984.

[59] K. Tamura, E. P. Ippen, H. A. Haus, and L. E. Nelson, "77-fs pulse generation from a stretched-pulse mode-locked all-fiber ring laser," Optics Letters, vol. 18, no. 13, pp. 1080-1082, 1993.

[60] F. O. Ilday, F. W. Wise, and T. Sosnowski, "High-energy femtosecond stretched-pulse fiber laser with a nonlinear optical loop mirror," Optics Letters, vol. 27, no. 17, pp. 15311533, 2002.

[61] F. O. Ilday, J. R. Buckley, W. G. Clark, and F. W. Wise, "Self-similar evolution of parabolic pulses in a laser," Physical Review Letters, vol. 92, no. 21, Article ID 213902, 4 pages, 2004.

[62] F. O. Ilday, J. R. Buckley, H. Lim, F. W. Wise, and W. G. Clark, "Generation of 50-fs, $5-\mathrm{nJ}$ pulses at $1.03 \mu \mathrm{m}$ from a wavebreaking-free fiber laser," Optics Letters, vol. 28, no. 15, pp. 1365-1367, 2003.

[63] O. E. Martinez, R. L. Fork, and J. P. Gordon, "Theory of passively mode-locked laser including self-phase modulation and group-velocity dispersion," Optics Letters, vol. 9, no. 5, pp. 156-158, 1984.
[64] S. Namiki, E. P. Ippen, H. A. Haus, and C. X. Yu, "Energy rate equations for mode-locked lasers," Journal of the Optical Society of America B, vol. 14, no. 8, pp. 2099-2111, 1997.

[65] C. Antonelli, J. Chen, and F. X. Kärtner, "Intracavity pulse dynamics and stability for passively mode-locked lasers," Optics Express, vol. 15, no. 10, pp. 5919-5924, 2007.

[66] N. G. Usechak and G. P. Agrawal, "Semi-analytic technique for analyzing mode-locked lasers," Optics Express, vol. 13, no. 6, pp. 2075-2081, 2005.

[67] B. G. Bale and J. N. Kutz, "Variational method for modelocked lasers," Journal of the Optical Society of America B, vol. 25, no. 7, pp. 1193-1202, 2008.

[68] Y. Chen, F. X. Kärtner, U. Morgner, et al., "Dispersionmanaged mode locking," Journal of the Optical Society of America B, vol. 16, no. 11, pp. 1999-2004, 1999.

[69] A. Komarov, H. Leblond, and F. Sanchez, "Quintic complex Ginzburg-Landau model for ring fiber lasers," Physical Review E, vol. 72, no. 2, Article ID 025604, 4 pages, 2005.

[70] H. Leblond, M. Salhi, A. Hideur, T. Chartier, M. Brunel, and F. Sanchez, "Experimental and theoretical study of the passively mode-locked ytterbium-doped double-clad fiber laser," Physical Review A, vol. 65, Article ID 063811, 8 pages, 2002.

[71] F. O. Ilday and F. W. Wise, "Nonlinearity management: a route to high-energy soliton fiber lasers," Journal of the Optical Society of America B, vol. 19, no. 3, pp. 470-476, 2002.

[72] D. Anderson, M. Lisak, and A. Berntson, "A variational approach to nonlinear evolution equations in optics," Pramana Journal of Physics, vol. 57, no. 5-6, pp. 917-936, 2001.

[73] E. N. Tsoy, A. Ankiewicz, and N. Akhmediev, "Dynamical models for dissipative localized waves of the complex Ginzburg-Landau equation," Physical Review E, vol. 73, no. 3, Article ID 036621, 10 pages, 2006. 

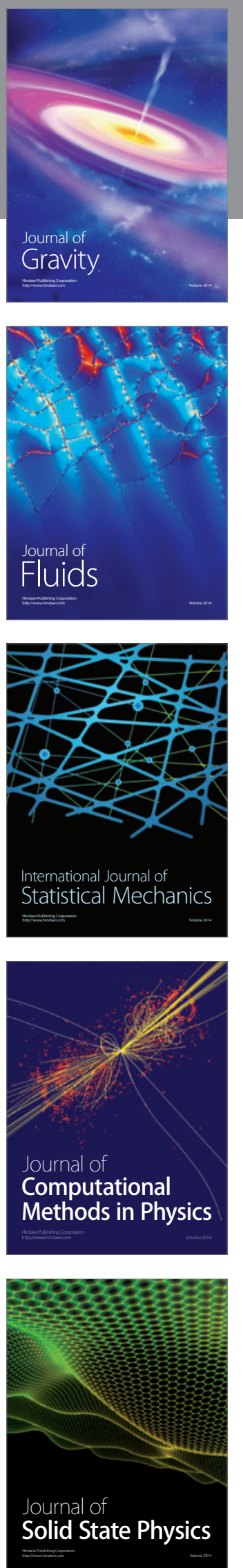

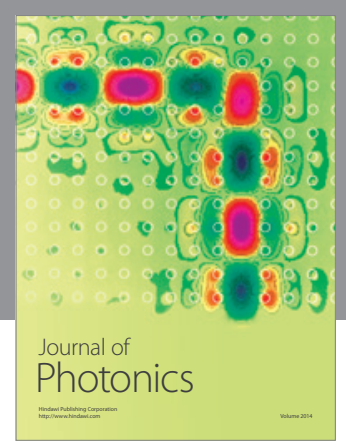

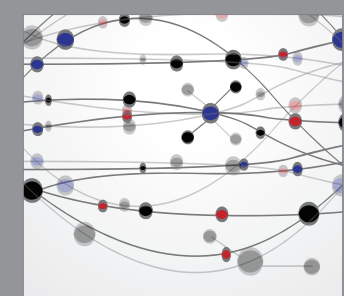

The Scientific World Journal
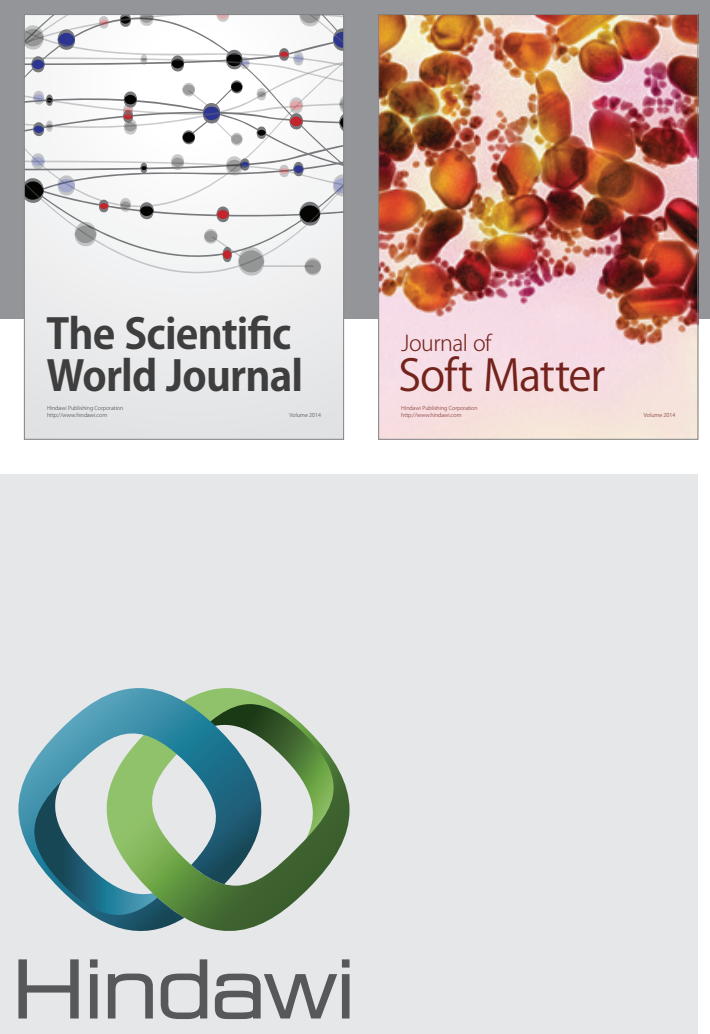

Submit your manuscripts at

http://www.hindawi.com
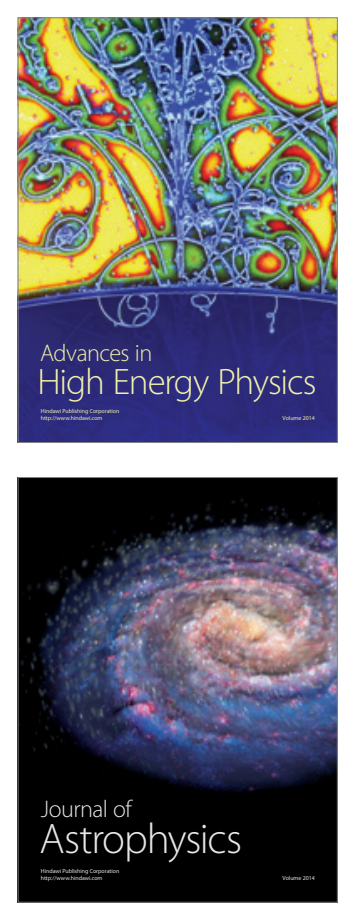
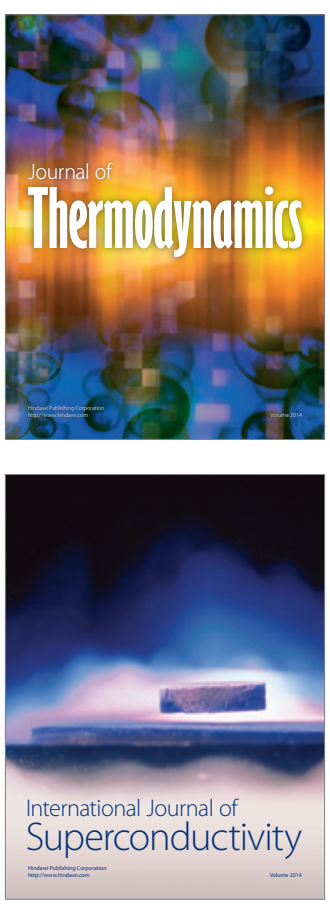
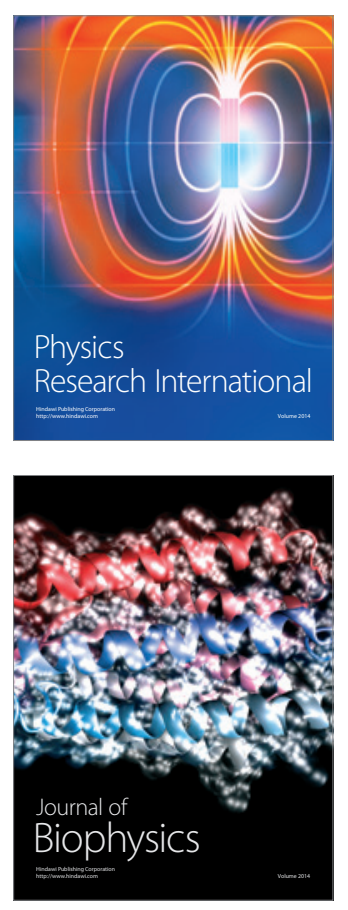
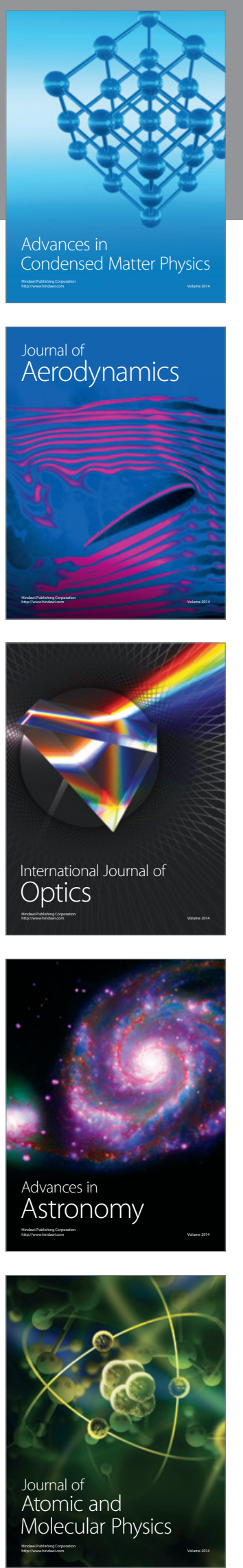